\section{Interactions between spatial and kinetic dimensions in movement aftereffect*}

\author{
CLAUDE BONNETt and VIVIANE POUTHAS \\ Laboratoire de Psychologie Experimentale et Comparée \\ Associé au C.N.R.S., University of Paris V, Paris $6^{\circ}$, France
}

In some conditions, the surface of the test figure on which one sees an aftereffect of movement does not fit with that part of the visual field previously adapted to a movement. Such an effect, called kinetic-figural effect, may be conceived of as resulting from an interaction between two perceptual systems, each one giving specific information: one for the kinetic aspects which are spatially defined, the other for the spatial relationship inside the visual field. Experiments are presented which indicated the validity of a "law of location" for a movement aftereffect, together with some effects of the spatial relationships between adapting and test fields upon the movement aftereffect.

When one fixates for some time a waterfall, the revolving disk of a record player, the pen trace of an electrorecording device, or any structured moving surface and then quickly changes one's gaze to a stationary surface, one will observe on it an apparent motion in the opposite direction which is called movement aftereffect. The movement aftereffect (MAE) is an entopic phenomenon, internally generated, which is projected upon some exteroceptive test field. But such a projection is not a condition necessary in order to observe this apparent movement, since it may be observed with the eyes closed or in a dark room. However, as far as the experimental conditions of observation of a MAE are concerned, the generally used situation is one in which, after having adapted part of the retina with a visual movement, the $S$ is requested to observe a nonmoving field (test field) on which $S$ will perceive this MAE. Most of the time the experiments are devoted to study the role of some variables of the adapting movement on the detection, the duration, or the velocity of the MAE observed on the nonmoving image of the adapting field. Little is known about the role of the structure of the test field on the MAE. The experiments reported in this paper are concerned with this problem.

It is generally admitted that a MAE observed agninst come tent fields is limited to that area of the test field corresponding to the retinal area previously adapted with an objective movement (Wohlgemuth, 1911; Holland, 1965; Masland, 1969). In the following, this result will be referred

\footnotetext{
*Part of this paper was presented at the meeting of the Psychonomic Society, San Antonio, November 1870 . The authors express their thanks to $H$. W. Leibowitz and R. Y. Sasson for so kindly being their native English-speaking consultants.

tAddress: 28, rue Serpente, Paris $6^{\circ}$, France.
}

to as the "law of location." This areal specificity of the MAE may, however, be true only with qualification of its statement as suggested by the results of Wohlgemuth (1911) and of Sekuler and Pantle (1967).

The areal specificity of an aftermovement which is suggested by the law of location is of considerable importance with regard to the explanation of the aftermovement. In effect, for many authors (Sutherland, 1961; Sekuler \& Ganz, 1963; Barlow \& Hill, 1963; Sekuler \& Pantle, 1967; Richards, 1971), the visual aftereffect of motion provides a phenomenon through which one may study a hypothetical movement-analyzing system in human Ss.

The general theoretical background for the hypothesis just mentioned is that two different systems could contribute to the perception of a visual motion. In one system, the physical motion of an object would be visually perceived on the basis of the changes in position of this object over time (displacement). Such a system would be mainly at work in an "object-relative situation" (Dunker, 1929; Wallach, 1968). However, it could also be at work in a "S-relative ituation" with, for instance, a long exposure time of the visual stimulus movement (Leibowitz, 1955; Bonnet, 1971). The other system, which is of interest in this paper, could be called "movement-analyzing system" (Richards, 1971). This system gives rive to a "sensation of motion" as such (Wallach, 1968), i.e., the perception of a hinetic property of a vinual object as contrasted with the perception of spatial (or better, spatiotemporal) relationships of this object relative to other objects of the visual field given by the other system. This movement-analyzing system would be mostly at work in "S-relative situations" (Dunker, 1929; Wallach, 1968) and would involve presumably the activity of the so-called "movement detectors" (Barlow \& Hill, 1963; Richards, 1971). Its stimulus would be presumably the movement of an image across the retina (Bonnet, 1971).

The MAE has been said (Gregory, 1966) to be a perfect example of a phenomenon in which a human $S$ may observe a pure movement in the visual field, without any correlative displacement. In effect, provided his attention will be directed towards this aspect, a $\mathbf{S}$ will describe a somehow paradoxical aspect of the MAE. After adaptation to a linear motion (waterfall effect), a $S$ will perceive an apparent movement in the reverse direction, but he will not observe any change in the position or in the spatial location of the previously moving stripes. After adaptation to a rotating spiral, an expansion (or a contraction) of the nonmoving test spiral will be perceived without any change in its perceived size and shape. In other words, these observations would indicate that a MAE does not affect the perceived spatial or figural relationships in the test field.

If one assumes that there are two distinct systems (movement and displacement) for the visual perception of a physical motion, it seems reasonable to relate the MAE to the movement-analyzing system as suggested above. Moreover, if the stimulus for this system is retinal, then the law of location for the MAE would only be a consequence of this fact. The paradoxical dissociation between perceived movement and perceived displacement in MAE would also be logical since each depends upon a different system.

The first three experiments presented below deal with the problem of the validity of the law of location, since the areal specificity which is implied in it is central for the interpretation of the mechanism of the MAE.

\section{PRELIMINARY OBSERVATIONS ${ }^{1}$}

While intended for an entirely different purpose, the basic situation for examination of the validity of the law of location was suggested by Goldstein (1958).

One may use as an adapting field a spiral that is rotating behind a sereen which has four slots arranged in the shape of a cross. In such a case, the adapting movement looks like four concentric "waterfalls." However, if the MAE of such a field is observed against an entire nonmoving spiral, Ss would describe it as affecting the whole test spiral. This observation, which is readily made, is in apparent contradiction with the law of location, since an areal spread of the MAE occurs in such a way that a MAE is 
also perceived in parts of the visual field which have not been previously adapted to the movement.

Three complementary observations will help in defining the phenomenon and in pointing out the fact that it may be due to some spatial properties of the test field. When using the same adapting field just described, one may observe different phenomena, depending upon the kind of test field used.

(1) An unstructured test field (nonmoving visual noise) gives rise to the observation of four MAEs located in those parts of the visual field which have been previously adapted. In such a case, the law of location holds.

(2) If the test field is the nonmoving image of the adapting field, a MAE is perceived only along the four slots; no MAE is detected against the homogeneous part of the test field between the slots, in spite of the fact that such a detection is possible for part of a homogeneous test field, as long as it corresponds to a previously adapted area.

(3) If the test field is the nonmoving image of the adapting field, but with the slots slanted by $45 \mathrm{deg}$ so that there is no corresponding part between the adapting and test fields, no MAE can be observed inside the four radii. However, some MAE can be observed against the homogeneous background corresponding to the previously adapted retinal area.

In other words, an areal spread may appear, which seems to invalidate the law of location, only when some figural properties of the test fields, or some spatial relationships between adapting and test fields are met. They are to be defined further. Before that, the question is raised: Did the reported result invalidate the law of location? Obviously, presented as it is up to now, such an observation seriously invalidates the areal specificity of a MAE assumed by the law of location. However, careful observations made in the same situation indicate that the areal spread of the MAE did not appear at the very beginning of the test phase. When tracking the phenomenon over time, it was first seen that an aftermovement was observed in areas corresponding to the adapted one. Then, after a few seconds, the areal spread was noticed on the whole nonmoving test spiral. In such situations, the observed MAE follows two successive phases. First, a "kinetic" phase is noticed during which the law of location holds. We propose to label it the "kinetic" phase because of the assumption that the areal specificity of a MAE (law of location) would be a property of the movement analyzing system. In other words, this assumption may be stated by saying that in "movement detectors," some mapping of the retinal fields would appear. Following this phase of areal specificity, an areal spread is noticed which may be labeled "figural" phase. The reason for such labeling is the assumption that the areal spread would result mostly from the figural properties of the test field.

Then, if, in situations where some areal spread of the MAE appears, this areal spread is always preceded by a phase (kinetic phase) during which some areal specificity can be noticed, the law of location is not essentially invalidated. In the following experiments, the problem of the appearance of two phases in such conditions will be investigated, together with examination of the effect of some factors of the adapting field.

\section{GENERAL PROCEDURE}

Except for special specifications mentioned later on, the general procedure of the experiments is the following one.

(1) Very sophisticated Ss were used. They had been trained in preliminary experiments in order to minimize the variability of their responses for the duration of the MAE in standard situations. At the same time, from trial to trial, the reliability of the responses improved, the duration of the reported MAE tended to increase, up to a stabilized performance. Presumably these variations of performance were due to the stabilization of the criterion of response: the $\mathbf{S}$ was instructed to indicate, by pressing a key, when he was absolutely sure that the MAE was over, i.e., when the test figure from the point of view of movement perception, looked like it was before the adapting period. The increase in mean duration of the MAE with replications may also be related to an increasing sensitivity to movement and to better fixation during both the adapting and the test periods.

From another point of view, it has been found that if the areal spread is easily observed by "naive" Ss, the distinction between the two phases is not so easy to obtain and especially the relative durations of these two phases.

(2) The adapting and test fields were the two channels of a Dodge-type tachistoscope. The $S$ observed monocularly with the preferred eye. Each field was $19 \times 15 \mathrm{~cm}$, and each was located at a distance of $60 \mathrm{~cm}$. The luminance level of each field was $10 \mathrm{~lx}$

During each trial, $S$ observed the movement of the adapting field for 30 sec while fixating a point located in the center of the moving field. This adapting movement was generally the rotating movement of a spiral inscribed inside a circle $11 \mathrm{~cm}$ in diam. At the end of the 30 -sec adapting period, the test field reappeared with a fixation point which was superimposed spatially on the fixation point of the adapting field. The task of the $\mathbf{S}$ consisted first in pressing on a key to indicate when the MAE was over. Eventually, when two phases appeared, the $S$ pressed on a first key to indicate the end of the first phase, and then on a second key to indicate the end of the second phase. A verbal description of the phenomenon was generally requested after these responses.

On the basis of preliminary experiments, an intertrial delay of 1 to 2 min was used since it has been observed that such a delay was long enough to eliminate the negative effects of massed presentations (see Holland, 1965).

\section{EXPERIMENT 1 : FACTORS IN THE ADAPTING FIELD FOR AN AREAL SPREAD}

The aim of this experiment was to look for the effect of some factors of the adapting field in the appearance of an areal spread and in the detection and duration of a first "kinetic" phase in such cases.

The test field presented a black background (B). In the middle of it, a circle of $11 \mathrm{~cm}$ diam was cut out, through which appeared an arithmetic spiral of seven throws. (Actually only 6.25 throws were seen.) This spiral was black (b) (2 mm wide) drawn on a white background $(w)$, so that the ratio of black/white (b/w) was about .3. This test field will be referred to as (B, b/w).

The adapting field presented, basically, the rotating movement of a four-throw arithmetic spiral. Its diameter was $11 \mathrm{~cm}$, and the ratio of white/black was equal to 1 . The speed of rotation was $120 \mathrm{rpm}$.

The adapting movement was seen only through angular sectors of a black mask located in front of the rotating spiral. The angular sectors, used instead of the rectangular slots of the preliminary observations, were cut from the center of the spiral, which was the fixation point. Each sector was $5.5 \mathrm{~cm}$ long (in Fig. 3 , see example of a field used in Experiment 3).

In the quantitative experiment, eight experimental conditions were presented, resulting from the combination of two variables. The first variable was the number of sectors through which $\mathbf{S}$ saw part of the adapting spiral: one, two, three, or four sectors were used, symmetrically placed in front of the adapting spiral except for the Condition 1 sector. The 


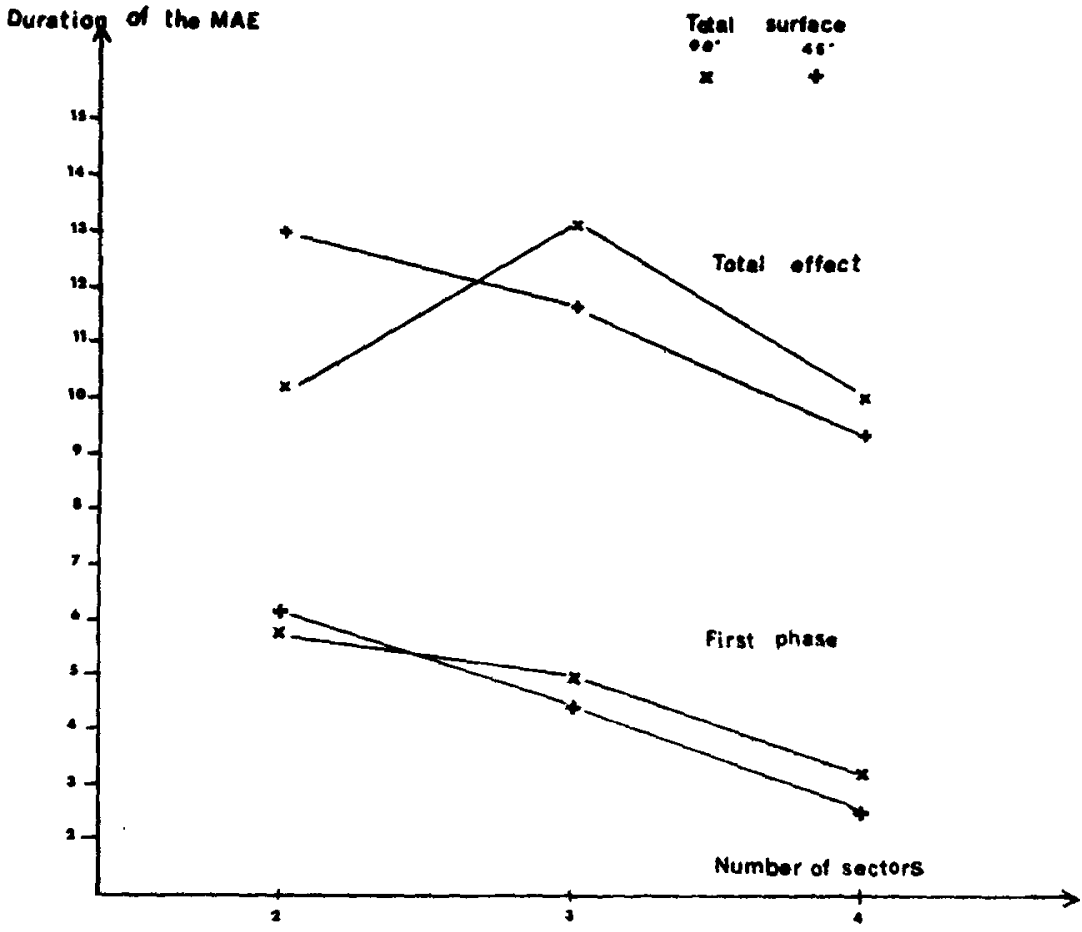

Fig. 1. Duration of the kinetic phase and total duration of the MAE as a function of the number of sectors of the adapting field.

second variable was the total angular surface defined as the sum of every angular slot; two total surfaces were used, 90 and 45 deg, e.g., a total surface of $90 \mathrm{deg}$ with four sectors means that each of the four sectors was $90 / 4=22.5 \mathrm{deg}$.

\section{Nominal Experiment}

A preliminary experiment was carried out with 10 naive Ss in order to test the generality of the qualitative observations made in such conditions. Ss were presented with the situation just decribed but only with the $90 \mathrm{deg}$ total surface. They were requested to describe verbally what they saw in the four different conditions. The $E$ never commented upon their responses except for asking them to be more precise. The questions (what was moving, where something was moving, and how) were asked only by using the Ss' own words.

One of the10 Ss was never able to report a MAE. None of the 9 other Ss had reported an areal spread for the one-sector condition. Seven of the 9 described, without any doubt, an areal sprear for the three other conditions. The responses of the 2 other $S s$ were impossible to categorize. Next, the Ss were presented with the test field for a short period (about $2 \mathrm{sec}$ ) and asked to describe what happened during this time. All 7 perceived a MAE restricted to the previously adapted area: the law of location held. In using again a time-free situation for the test period, the 7 Ss, when instructed properly, succeeded in describing two successive phases in the effects.

Further observations made since that time indicate that very few Ss never succeed, after some training, in being able to describe two successive phases in situations where some areal spread of the MAE appeared. Very likely, those who never succeeded are also those who perceived very faint and short MAEs in normal conditions, if they perceived any. Consequently, one may consider that the areal spread does not invalidate the law of location; the test period. verbatim comments and more reliable measurements of any duration of the phenomenon with naive Ss points to the necessity of using sophisticated Ss in quantitative experiments.

\section{Quantitative Experiment}

This experiment was devoted to the measurement of the duration of the two phases for different conditions. The Ss considered as sophisticated were the present authors. After preliminary trials to check the reliability of the qualitative description of the phenomena, four experimental trials were presented for each of the eight experimental conditions, run in a random order. The task of the $S$ was to press on one key at the end of the "kinetic" phase, if any. Actually, the Ss pressed on the it only restricts its validity to a part of

The difficulty in obtaining reliable key when they saw the MAE was no longer restricted to the previously adapted area. They pressed a second key when they no longer detected MAE.

The results are presented in Fig. 1. The one-sector condition $(45$ or 90 deg total surface) has not been reported in this graph since no areal spread is ever noticed in such conditions.

In Fig. 1, the effect of the total surface does not appear significant. The duration of the "kinetic" phase decreases with the number of sectors, so it is for the total duration (kinetic + figural phases) in such a way that the duration of the "figural" phase appears to be about constant. Statistical tests are not reliable because of the small number of observations. However, the standard deviations are quite low and do not vary systematically with the conditions. The mean standard deviation for the "kinetic" phase was .76 sec for C.B. and .55 for V.P.; for the "figural" phase, the mean standard deviation was .88 for C.B. and 1.1 for V.P.

It was observed that the greater the number of sectors, the shorter the time during which the law of location holds (i.e., duration of the "kinetic" phase), but at the same time the shorter the total duration of the MAE, in spite of the fact that the total surface is constant. These results indicated clearly that the two phases in the effect were measurable in a reliable way as long as the requirement of sophistication of the Ss was satisfied. No answer could be given to the question of whether the change from the "kinetic" to the "figural" phase is abrupt or progressive.

Having found no areal spread with only one adapting sector of either 45 or $90 \mathrm{deg}$, a new experiment was carried out in order to determine if a larger size sector would increase the observation of some areal spread.

Effect of the Size of One Sector

The test field used in this experiment was the same as in the preceding one: $(B, b / w)$. The adapting field was different. These differences were not considered as critical for the effect and have been introduced for another purpose. The adapting spiral was a seven-throw arithmetic spiral identical to the test spiral. Its speed of rotation was $120 \mathrm{mpm}$. It was presented behind an angularly slotted screen. Three experimental conditions were chosen according to the angular size of the slots: 90,135 , and $180 \mathrm{deg}$.

The Ss were three students in psychology who were considered to be well trained with regard to this kind of situation. After preliminary trials, four experimental trials were given for 
Table 1

Duration of the Kinetic Phase and of the Total MAE (Mean in Seconds)

\begin{tabular}{llll}
\hline Size & $\begin{array}{c}90 \\
\text { Deg }\end{array}$ & $\begin{array}{c}135 \\
\text { Deg }\end{array}$ & $\begin{array}{c}180 \\
\text { Deg }\end{array}$ \\
\hline $\begin{array}{l}\text { Kinetic } \\
\text { Phase }\end{array}$ & - & 5.63 & 3.96 \\
$\begin{array}{l}\text { Total } \\
\text { Duration }\end{array}$ & 11.15 & 17.30 & 15.57 \\
\hline
\end{tabular}

every size of the angular slot, presented in a random order. S's task was to indicate, by pressing a key, the end of the "kinetic" phase, if any, and to indicate, by pressing a second key, the end of the total MAE. Mean numerical results are presented in Table 1.

When the size of the angular slot was 90 deg, no areal spread was ever perceived, as seen in the experiment (1b) for two other Ss. With 135 and $180 \mathrm{deg}$, at every trial, for every $S$, an areal spread was detected which never appeared at the very beginning of the test period. These results indicate that very likely increasing the size of the angular sector would decrease the duration of the "kinetic" phase (i.e., the duration of the phase during which the law of localization holds) and would also reduce correlatively the total duration of the MAE. They also provide another indication that the duration of the "figural" phase could be about constant. Very likely one may conclude from these results that for a one-sector condition, there exists a critical size of the sector for the appearance of an areal spread effect.

These results raise a question. In effect, it is known that increasing the size of the adapting field in standard situations increases the duration (total) of the MAE (Freud, 1964). In our situation, it was seen to the contrary that increasing the size of the adapting field between 135 and $180 \mathrm{deg}$ could decrease the total duration of the MAE. No more can be said about this result than to assume, if such a difference could be found to be significant, that the areal spread effect may interfere with the "normal" process of the MAE.

\section{EXPERIMENT 2: AREAL SPREAD}

AND VIVIDNESS OF THE MAE

If the observation of two successive phases in the areal spread effect allows us to consider that the law of location is still valid, one may wonder what factor may explain the relative duration of these two phases.

In standard conditions of observation of a MAE, many authors (see Holland, 1965) have described systematic variations of vividness, intensity, or velocity during the course of the test period. There are some indications that the three variables are related. In any event, such a result was perfectly illustrated in the data of Taylor (1963). This author, using a tracking technique of nulling the MAE, found that the decrease of the velocity of the MAE was negatively accelerated, first very rapidly, and later, more slowly. The two phases in the decrease of the velocity or the vividness of the MAE could be described in this way: first the MAE is very vivid and collapses rapidly, then it is less distinct and decreases more slowly. The relative duration of the two phases may be measured reliably.

One may assume that, when conditions are met in which an areal spread of the MAE appears, as long as the MAE is vivid enough (first phase), the law of location remains valid. In other words, the duration of the first phase of vividness would be equal to the duration of the "kinetic" phase for the same adapting movement.

To test this hypothesis, it is necessary to use a test field which does not give any areal spread for measuring just the relative duration of the two phases of vividness, and then to compare these durations, for the same adapting field, with the durations of the "kinetic" and "figural" phases obtained when the test field gives rise to an areal spread.

The test field chosen for this experiment was a nonmoving visual noise which, as reported in the preliminary observations, never gave the detection of an areal spread.

Part of the conditions used in Experiment 1b also served in this experiment for the adapting field. We restricted the experiment to conditions of two, three, and four sectors with a total surface of $45 \mathrm{deg}$. Ss and other conditions were also identical to Experiment 1b.

After preliminary trials, the Ss could notice the difference in vividness of the two phases of a MAE observed against a visual noise test field. During the experiment, they were instructed to press a first key in order to indicate when the MAE started to be less vivid (end of the first phase) and to press a second key when they no longer perceived the MAE (end of the second phase).

The mean results are presented in Fig. 2, together with the results of the corresponding conditions in Experiment 1b. The variability was of the same order as in Experiment $1 b$.

While, phenomenally, the two test

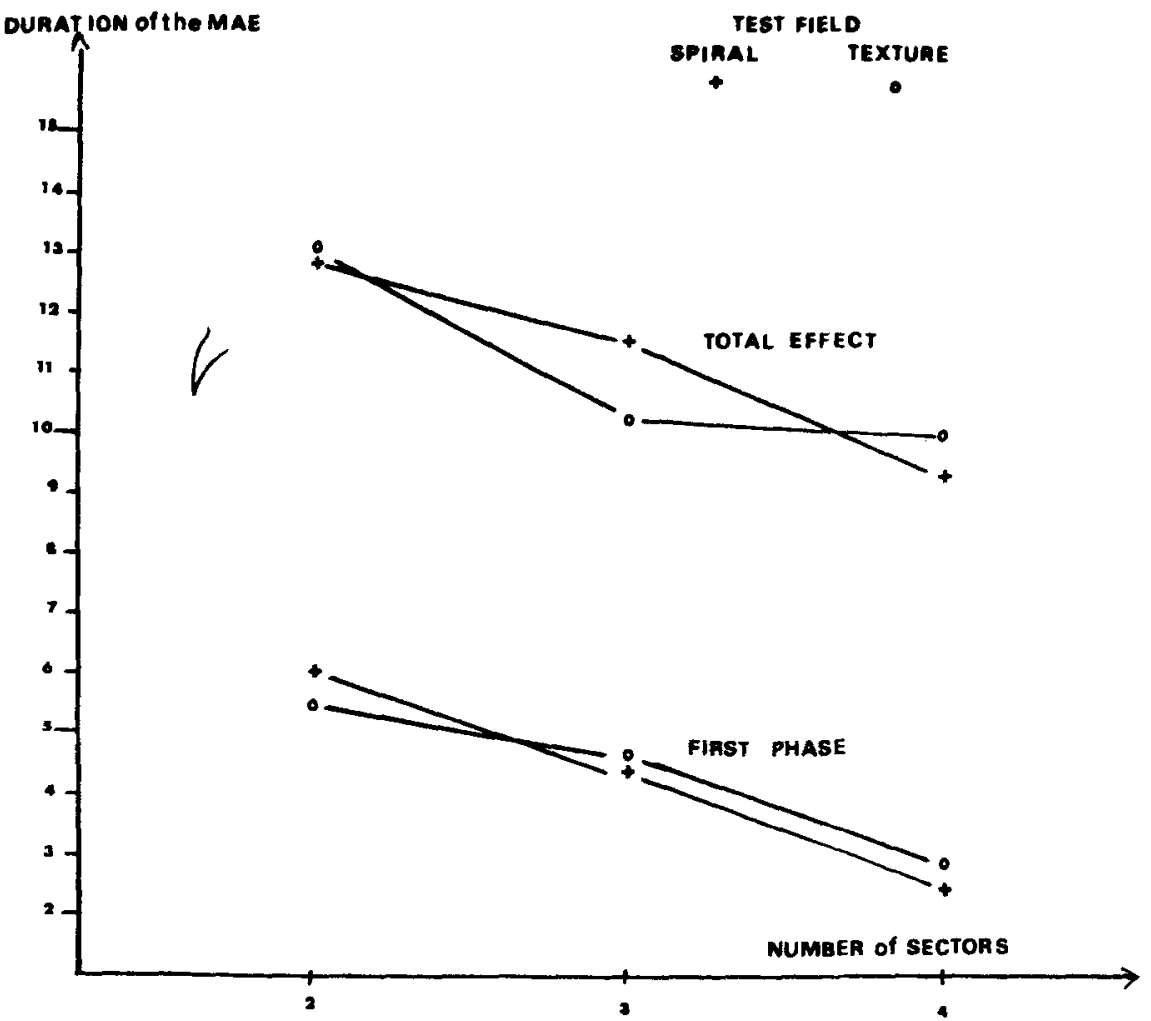

Fig. 2. Duration of the first phase of vividness of a MAF and total duration as a function of the number of sectors of the adapting field compared with the duration of the kinetic phase (see text). 

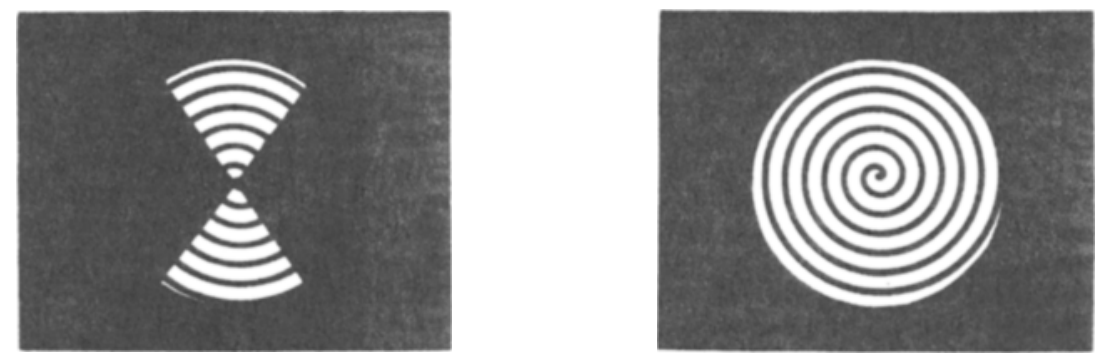

A

Fig. 3. Adapting field (A) and test field (T) used in Experiment 3. (The test field was used only in preliminary trials not reported in the text.)

fields used in these two experiments produced different perceptions, and while different phenomena were measured, the results appeared quite similar. They indicate, first, that the relative duration of the two phases of vividness could be measured reliably and, second, that the above assumption has some likelihood: the law of location seems to remain valid as long as the vividness of the MAE is strong enough.

Obviously these data alone are not sufficient to prove the assumption but did encourage further research. These two first experiments have given indications about some factors in the adapting field which may vary the appearance and duration of an areal spread. The second experiment suggested the reason why the law of location remained valid for part of the test period, even when some areal spread appeared in some situations.

\section{EXPERIMENT 3: AREAL SPREAD AND STRUCTURE OF THE TEST FIELDS}

It has been shown in the preliminary observations that an areal spread of the MAE may be dependent upon the figural structure of the test field. For instance, for the same adapting field, the areal spread appeared when the MAE wos projected upon a test spiral and not when it was projected upon a nonmoving visual noise. Very likely, if come figural characteristic of the test field are necessary in order to obtain an areal spread, it is also necemary to have some degree of configural similarity between adapting and teat fields. The role of the later variable will be examined further in Experiment 4. However, in the next experiment, this configural similarity will also be at work, since adapting and teet fields presented basically the same spiral.

The experiment was done in order to test how the figural characteristics of the test field, as presumably part of the configural similarity, may modify the areal spread generally observed in such situations.

The adapting field was a seven-throw arithmetic spiral $(B, b / w)$, observed for $30 \mathrm{sec}$ through angular sectors. The screen had two angles, $60 \mathrm{deg}$ each, symmetrically placed with regard to the vertical, through which appeared part of the moving spiral rotated at $120 \mathrm{rpm}$.

The test field was basically the same nonmoving spiral. It was presented in four different conditions. In a first set of conditions, we superimposed on the spiral a drawing of two or three diameters; in both cases, two of them defined boundaries of two angles identical in size and orientation to the angles of the adapting field, i.e., with two diameters, the test spiral had two sectors of $60 \mathrm{deg}$ each and two of $120 \mathrm{deg}$, while with three diameters, the test spiral had six equal sectors. The second variable, crossed with the first, was the "color" of these diameters; they could be black or white. The width of these diameters was equal to the width of the throw of the spiral $(2.5 \mathrm{~mm})$. Then the four conditions of the experiment were presented in a random order to five sophisticated Ss. After preliminary trials, they all had four trials for every condition. During preliminary trials, the Ss' task was to detect if they saw any areal spread in any of the conditions. When their responses had stabilized, during the experimental session, they were to press a key at the end of the "kinetic" phase, if any, and to press another key at the end of the MAE. The results are presented in Table 2.

Analysis of variance of the duration of the total MAE indicated a nonsignificant $(F<1)$ variation of this duration as a function of the number of diameters in the test field. A slight but nonsignificant ( $F=3.25, \mathrm{df}=1,4$ ) variation was found for the "color" of these diameters, the tendency being to have longer duration with a black diameter than with a white one.

When considering the situation with regard to the detection of an areal spread, a very strong dichotomy appears. This areal spread appeared only with two diameters and never for three. In the latter situation, the MAE always remained restricted to that part of the visual field which had been previously stimulated: the law of location held. For the two-diameter situation, like in a diameter-free situation, an areal spread was noticed by the Ss and, in addition, the test figure appeared to be affected in its shape: instead of perceiving a motion inside a circle, the $S$ s reported the spiral as stretched up and down along its vertical axis so that it appeared more or less oval in shape. It must be recalled that the MAE was an expansion, i.e., a centrifugal motion. This observation is very important, since it is one of the rare instances in which a MAE may affect the apparent shape of the test field.

The same conditions with two white diameters was presented to eight naive Ss for checking the appearance of the

Table 2

Duration (in Seconds) of the MAE and of the "Kinetic Phase" for Diffexent Structures of the Test Field

\begin{tabular}{|c|c|c|c|c|c|c|c|}
\hline \multirow[b]{3}{*}{ Ss } & & \multicolumn{4}{|c|}{ Total Duration } & \multicolumn{2}{|c|}{ Kinetic Phase } \\
\hline & & \multicolumn{2}{|c|}{ Two Diameters } & \multicolumn{2}{|c|}{ Three Diameters } & \multicolumn{2}{|c|}{ Two Diameters } \\
\hline & & White & Black & White & Black & White & Black \\
\hline VP & $\begin{array}{l}\text { Mean } \\
\text { SD }\end{array}$ & $\begin{array}{r}17.34 \\
1.09\end{array}$ & $\begin{array}{r}18.32 \\
1.64\end{array}$ & $\begin{array}{r}15.12 \\
1.62\end{array}$ & $\begin{array}{r}18.33 \\
1.47\end{array}$ & $\begin{array}{l}3.64 \\
0.50\end{array}$ & $\begin{array}{l}\mathbf{3 . 3 4} \\
\mathbf{0 . 4 7}\end{array}$ \\
\hline CB & $\begin{array}{l}\text { Mean } \\
\text { SD }\end{array}$ & $\begin{array}{r}13.97 \\
1.23\end{array}$ & $\begin{array}{r}17.34 \\
1.30\end{array}$ & $\begin{array}{r}14.38 \\
1.09\end{array}$ & $\begin{array}{r}17.91 \\
2.07\end{array}$ & $\begin{array}{l}3.19 \\
0.45\end{array}$ & $\begin{array}{l}4.70 \\
0.32\end{array}$ \\
\hline $\mathbf{A M}$ & $\begin{array}{l}\text { Mean } \\
\text { SD }\end{array}$ & $\begin{array}{r}13.08 \\
1.82\end{array}$ & $\begin{array}{r}11.45 \\
1.10\end{array}$ & $\begin{array}{r}12.23 \\
1.80\end{array}$ & $\begin{array}{r}13.05 \\
0.92\end{array}$ & $\begin{array}{l}3.82 \\
0.51\end{array}$ & $\begin{array}{l}2.97 \\
0.30\end{array}$ \\
\hline $\mathbf{D T}$ & $\begin{array}{l}\text { Mean } \\
\text { SD }\end{array}$ & $\begin{array}{r}12.80 \\
2.26\end{array}$ & $\begin{array}{r}14.88 \\
1.59\end{array}$ & $\begin{array}{r}14.23 \\
1.39\end{array}$ & $\begin{array}{r}13.54 \\
1.45\end{array}$ & $\begin{array}{l}2.91 \\
0.53\end{array}$ & $\begin{array}{l}3.74 \\
0.42\end{array}$ \\
\hline DV & $\begin{array}{l}\text { Mean } \\
\text { SD }\end{array}$ & $\begin{array}{r}13.16 \\
2.72 \\
\end{array}$ & $\begin{array}{r}11.05 \\
2.26\end{array}$ & $\begin{array}{l}9.83 \\
0.13\end{array}$ & $\begin{array}{r}13.81 \\
0.89\end{array}$ & $=$ & $\begin{array}{l}4.41 \\
1.78\end{array}$ \\
\hline
\end{tabular}


phenomenon. They all described a change of form in the test field, using most often the comparison of an "egg form." In another experiment, we have checked the appearance of this deformation with a contracting MAE, i.e., a centripetal motion. In that case, the spiral was flattened and appeared inscribed in an oval with a horizontal great axis.

In other words, the conclusion may be drawn from this experiment that increasing the configural similarity of the test field with the adapting field allows not only an areal spread of the MAE, but also may suppress it: paradoxical aspect in inducing a change in shape coherent with the perceived movement. Such a phenomenon did not appear when three diameters were placed in front of the test spiral. One may think that in the three-diameter situation, some symmetry was reintroduced in the figure which presented the areal spread. However, some observations have been done, using as an adapting field of two angular sectors of $90 \mathrm{deg}$ each. Two different test fields have been used: one was the entire spiral, the second was the same entire spiral with two diameters corresponding to the sectors of the adapting field. In the latter case, the test figure was entirely symmetrical, since each of the four angles delimited by the black diameters was of $90 \mathrm{deg}$. The MAE observed against the entire spiral manifested an areal spread which did not modify the apparent shape of this spiral. However, when observed against the two-diameter test field, not only is an areal spread noticed, but there is also a change in the apparent shape: the spiral looked stretched up and down along an axis corresponding to the middle of the previously stimulated angles. In the case of the three-diameter condition, one may assume that another factor explained why no areal spread was noticed. Presumably, superimposing three diameters on the test spiral had reduced what may very roughly be called the "figural prägnanz" of the test field.

\section{EXPERIMENT 4:}

\section{CONFIGURAL SIMILARITY}

It has been said in introducing the preceding experiment that very likely some degree of configural similarity between adapting and test fields was necessary in order to be "able to observe an aredl spread of the MAE. The effect of configural similarity and of the structure of the test field were hard to distinguish in such an experiment. Consequently, it was chosen to study their effects in the absence of any areal spread.

If MAE reflects quite directly the activity of the "movement detectors" (Barlow \& Hill, 1963), then out of the areal specificity expressed by the law of location there is no reason why, in a standard situation, the MAE has to interfere with the spatial structure of the test field. From this point of view, the areal spread reported in the preceding experiments was not necessarily critical with regard to such a hypothesis. However, some data exist which indicate that this hypothesis may be wrong. During the last century and at the beginning of this one (see Wohlgemuth, 1911), the situations in which no distal test field was presented (eye closed or dark room) showed that if a MAE could be perceived, it generally lasted less time. From our own experience, the same conclusion seemed to hold when the distal test field was homogeneous, like a white cardboard field.

These observations and others which are now being investigated indicated that some heterogeneity of the distal test field facilitated the observation of a MAE and increased its reported duration. As far as these minimal conditions of heterogeneity of the distal test field are met, the critical question is to determine if any change in the reported duration of the MAE may occur as a function of changes in the structure of the test field. Operationally, this problem will be examined in the next experiment, together with the problem of the role of the configural similarity between adapting and test fields on the duration of the reported MAE.

While a very clear definition of the configural similarity is hard to state a priori, empirical situations will illustrate what could be understood with this concept.

The aim of the next experiment was to test the effect of the configural similarity between adapting and test fields upon the duration of the MAE, together with the effect of the structure of the test field in situations in which no areal spread may appear.

Four test fields were used: $\mathrm{T}(\mathrm{B}, \mathrm{b} / \mathrm{w})$-On a black background (19 $x \quad 15 \mathrm{~cm}$ ) (B) was presented a seven-throw arithmetic black spiral (b) drawn on a white disk (w) $11 \mathrm{~cm}$ in diam. The thickness of the stroke was $2 \mathrm{~mm}$, so that the ratio of black/white was .285. $T(W, w / b)$-This test field was the reverse of the preceding one, a white background (W), with a seven-throw arithmetic white spiral (w) drawn on a black disk (b). The ratio of black/white is $1 / .285=3.5$. $T(R, C C)-A$ white background (19 x $15 \mathrm{~cm}$ ) was filled with a drawing of concentric circles (CC) with a separation of $.5 \mathrm{~cm}$ superimposed with the drawing of radii ( $R$ ), $22.5 \mathrm{deg}$ of separation. The thickness of the strokes was $1 / 3 \mathrm{~mm}$. T(VN)-The total background $(19 \times 15 \mathrm{~cm})$ was filled with a "nonmoving visual noise" (VN), made of black elements sprinkled on the white background (Letraset texture).

The first two of these test fields were successively used as adapting fields $[(B, b / w)$ and $(W, w / b)]$. In each case, the speed of rotation was $120 \mathrm{rpm}$, providing an expansion MAE.

Two well-trained Ss (the authors) made eight duration estimates in each of the eight situations. The results are reported in Table 3.

Overall, it is seen that the $(W, w / b)$ adapting spiral gave longer MAE durations. An analysis of variance has been made separately for each $S$, indicating that this comparison was significant for both $\mathrm{Ss}[\mathrm{df}=1,7$; F(V.P. $)=23.1, p<.005 ; F(C . B)=$. $12, p<.02]$. In the same way, these analyses of variance indicated the significant effect of the nature of the test field [ $\mathrm{df}=3,21 ; \mathrm{F}$ (V.P.) $=19.8$, $\mathrm{p}<.0005 ; \mathrm{F}$ (C.B.) $=11.1, \mathrm{p}<.005]$ and of the Test by Adapting Fields interaction $[\mathrm{df}=\mathbf{3 , 2 1} ; \mathrm{F}$ (V.P.) $=\mathbf{1 0 . 9}$, $\mathrm{p}<.0005 ; \mathrm{F}(\mathrm{C.B})=9.6,. \mathrm{p}<.0005]$. It is seen that the differences in durations for (R, CC) and (VN) were not significani [df $=1,21 ; F(V . P)=$. 1.6, n.s.; $F(C . B)<1$.$\} , while these$ two durations were significantly lower than the durations for the two spiral test fields [ $d f=1,21 ; F(V . P)=$.38.42 , p $<.0005 ; \quad F(C . B)=$.27.35 , $p<.0005]$. While the comparison of the durations for the two test spirals was not very significant [ $d f=1,21$; $F($ V.P. $)=5.79, p<.02 ; F(C . B)=$. 0.35 , n.s. $]$, the comparison test spiral identical to the adapting one $[A$ : $(B, b / w), T:(B, b / w)$ and $A:(W, w / b)$, $\mathrm{T}:(\mathrm{W}, \mathrm{w} / \mathrm{b})]$ against the "negative" case $[A:(B, b / w), T:(W, w / b)$ and $A$ : $(\mathrm{W}, \mathbf{w} / \mathrm{b})$, $\mathrm{T:}(\mathrm{B}, \mathrm{b} / \mathrm{w})]$ was highly significant for both $\mathrm{Ss}$, with an overall mean of $19.80 \mathrm{sec}$ for identical spirals and $16.93 \mathrm{sec}$ for "negative" spirals I $\mathbf{d f}=1,21 ; \quad F(V . P)=$.24.29 , $\mathrm{p}<.0005 ; F($ C.B. $)=9.53, \mathrm{p}<.005]$.

As can be seen from the results of this experiment, some configural similarity between adapting and test fields increased the duration of the MAE. However, the fact that "negative" test spirals gave longer MAEs than other kinds of structure of the test field different from the adapting field may also indicate that, disregarding the configural similarity between adapting and test field, some test fields may give longer MAEs because of their own structure. It must be noticed that such a seven-throw arithmetic spiral, seen as nonmoving previous to any moving adaptation, gives the perception of some "dynamic", effects like those 
Table 4

Durations (in Seconds) of the MAE as a Function of the Test Field for a Rotational Adapting Movement

\begin{tabular}{|c|c|c|c|c|c|}
\hline Ss & Test & $(B, b / w)$ & $(w, w / b)$ & $(\mathrm{R}, \mathrm{CC})$ & $(\mathbf{V N})$ \\
\hline $\mathbf{V P}$ & $\begin{array}{l}\text { Mean } \\
\text { SD }\end{array}$ & $\begin{array}{r}16.80 \\
0.82\end{array}$ & $\begin{array}{r}17.08 \\
1.68\end{array}$ & $\begin{array}{r}13.79 \\
1.39\end{array}$ & $\begin{array}{r}24.00 \\
2.43\end{array}$ \\
\hline $\mathrm{CB}$ & $\begin{array}{l}\text { Mean } \\
\text { SD }\end{array}$ & $\begin{array}{r}17.39 \\
1.88\end{array}$ & $\begin{array}{r}16.77 \\
0.88\end{array}$ & $\begin{array}{r}15.05 \\
2.29\end{array}$ & $\begin{array}{r}25.59 \\
1.56\end{array}$ \\
\hline
\end{tabular}

reported by Mackay (1967) with concentric circles. The duration of the MAFs seen upon such a test field could be overestimated because these "dynamic" effects would make the end of the MAE more difficult to detect. From another point of view, the structure of the test figure, as such, may be more relevant for the detection of very faint MAEs. Our data are not decisive from this point of view.

In order to examine this point, a new experiment was performed, using the same four test fields. The adapting field presents the rotational movement of a "visual noise" with elements more discriminable than the elements of the test field "VN." The speed of rotation was $30 \mathrm{rpm}$ in order to maximize the duration of the MAE similar to that in the preceding experiment. The same two Ss took part in the experiment, in which eight trials for every test field were presented in a random order. The results are given in Table 4.

Again, one observes a significant increase of the duration of the MAE when the configural similarity between adapting and test field was maximum (test: VN) [df $=1,21 ; F(V . P)=$.193.3 , p $<.0005 ;$ F(C.B.) $=174.44$, p <.0005]. However, when the test field was made of a nonmoving spiral, the duration of the MAE was longer than it was with a less "dynamic" test field (R, CC) $[\mathrm{df}=1,21 ; \mathrm{F}$ (V.P.) $=$ 21.2, $\mathrm{p}<.0005 ; \mathrm{F}(\mathrm{C.B})=$.6.8 , $\mathrm{p}<.02]$.

Consequently, if, as shown in the preceding experiment, the configural similarity between adapting and test fields increased the duration of the perceived MAE, at the least some dynamical properties of the test field itself could also increase the duration of the perceived MAE projected on it.

\section{CONCLUSION} stimulating with regard to the explanation of the movement aftereffect. First, the areal specificity of the hypothetical movement-analyzing system as expressed by the law of location can be considered as valid. Secondly, some interactions have been noticed between the MAE and the
Two main results appear rather

structure of the test field on which it is projected. Two kinds of interactions, while related, have been observed: an increase in the reported duration of the MAE with incresse in the configural similarity between adapting and test fields and an areal spread of the MAE which may be considered as a partial lack of areal specificity of this phenomenon.

The latter phenomenon could be called "kinetic-figural effect" in MAE. Such a labeling (kinetic) points out the fact that no areal spread has ever been noticed at the very beginning of the test period of the MAE during which the law of location holds. In effect, this law has to be considered as one of the most important characteristics of the movement-analyzing system devoted to the perception of the kinetic properties of visual stimulation. From another point of view, the effect is also labeled "figural," since the areal spread appearing during the second phase of the test period seems to be related to the figural relationships in the test field.

Analysis of these figural relationships based on our empirical situations indicate conditions for the appearance of an areal spread. First of all, the adapting field must be spatially and figurally part of the test field, the last one having a high degree of structure. We have never noticed any areal spread when the MAE is observed against a "visual-noise" test field, but it is possible in some conditions to observe it in using the test field made of concentric circles and radii $(R, C C)$. However, the best situation for observing an areal spread seems to be when a high degree of configural similarity exists between adapting and test fields.

The role of the structure of the test

Table 3

Durations (in Seconda) of the MAE for Different Combinations of Adapting and Test Fields

\begin{tabular}{lllrrrr}
\hline Adapting & & Test & $(B, b / w)$ & $(w, w / b)$ & $(R, C C)$ & $(V N)$ \\
\hline & S VP & Mean & 17.17 & 11.53 & 11.38 & 12.67 \\
(B, b/w) & & SD & 4.50 & 1.42 & 0.86 & 1.51 \\
& S CB & Mean & 18.84 & 17.26 & 17.29 & 16.85 \\
& & SD & 1.41 & 1.42 & 1.26 & 1.07 \\
& S VP & Mean & 18.24 & 20.18 & 14.46 & 15.13 \\
(W, w/b) & & SD & 1.27 & 2.28 & 1.58 & 1.67 \\
& \multirow{3}{*}{ S CB } & Mean & 20.72 & 23.06 & 18.01 & 18.34 \\
& & SD & 2.81 & 1.61 & 1.39 & 2.12 \\
\hline
\end{tabular}

field and of its figural similarity with the adapting field indicated that if MAE reflects a "temporary imbalance of the maintained discharges of cells responsive to motion in opposite directions [Barlow \& Hill, 1963, p. 1346]," this mechanism would not be sufficient to explain the behavioral responses to a MAE. Tentatively, it may be admitted that such an activity in movement detectors is basic for perception of a MAE. However, some interaction may also appear between the perceived movement based upon such an activity and the figural information simultaneously picked up by the organism during the same test period.

In other words, the proposed explanation for the appearance and the variation of the kinetic-figural effect in MAE must assume that two kinds of information are integrated along separate channels in the organism. The two systems postulated in the very beginning of the present paper could be the basis for these two channels. In effect, it is reasonable to think that the displacement analyzing system which is devoted to the analysis of spatiotemporal relationships in the visual field may be involved in the perception of the figural characteristics of the adapting and test fields. Then a $S$ adapted in such a way as to perceive a MAE affecting only part of the test field will have two kinds of information. The first is movement information given through a spatially defined channel of motion sensitivity-he will perceive a MAE according to the law of location, the speed (or vividness), and the duration of the adapting movement. But at the same time, the $S$ will receive some exteroceptive information about the figural characteristics of the test field. The test field (the spiral for instance) is perceived as stable and not spatially affected by the movement which is projected on it-the spiral is still a regular one and still inscribed inside a regular circle. In other words, the $s$ experienced a paradoxical dissociation between the perceived movement and the perceived stability of the spatial relationships of the field inside which he perceives such a movement. This experience is paradoxical with regard to a cognitive model of such a situation, which assumes that any physical movement 
would imply some displacement or some change in the spatial relationships. In effect, if the perception of the two aspects (kinetic and figural) is paradoxical during the so-called "kinetic phase," during the figural phase the $S$ experienced a combination of the two kinds of information which appeared to be much more in agreement with the cognitive model. The best example of such a solution was for a two-sector adapting field and two-diameter conditions of the spiral test field. With such a high degree of configural similarity between adapting and test field, the $\mathbf{S}$ reported that the apparent shape of the test field was affected by the perceived movement in a way which could be predicted on the basis of a physical movement.

While the proposed formulation looks too "cognitivistic," the effect seemed to us rather perceptual. First of all, the reported effects were very stable for sophisticated Ss. The main problem in experimenting with them was that the duration of the kinetic phase may be rather short as compared with the total duration of the MAE, and an untrained $S$ may miss it. From our own experience, we have never noticed a situation in which the duration of the kinetic phase was undetectable. The presence of a kinetic phase preceding any experience of an areal spread is of importance with regard to the explanation of the effect, since, for us, it indicated that the two kinds of information (kinetic and figural) are coded separately and interacted only later inside the visual system.

\section{REFERENCES}

BARLOW, H. B., \& HILL, R. M. Evidence for a physiological explanation of the waterfall phenomenon and figural after-effects. Nature, 1963, 200 . 1345-1347.

BONNET, C. Les mécanismes de la perception de la vitesse d'un mouvement visuel. Bulletin de Psychologie, 1971, 24, 415-417.

DUNKER, $K$. Über induxierte Bewegung. Psychologische Forschung, 1929, 12. 180-259.

FREUD, S. L Duration of the spiral after-effect as a function of retinal size. retinal place, and hemiretinal transfer. Perceptual \& Motor Skills, 1964, 18. 47-53.

GOLDSTEIN, A. G. On the after-effects of the waterfall and spiral illusions. American Joumal of Psychology, 1958. A1, 608-609.

GREGORY, R. L. Eye and brain. London: World University Library, 1966.

HOLLAND, H. C. The spiral after-effect. London: Pergamon Press, 1965.

LEIBOWTTZ, H. W. Effect of reference lines on the discrimination of movement. Journal of the Optical Society of America, 1956, 49. 829-830.

MACKAY, D. N. Ways of looking at perception, In $\mathrm{w}$, Wathen-Dunn (Ed.) Models for the perception of speech and visual form. Cambridge, Mass: M.I.T. Press, 1967.

MASLAND, R. H. Visual motion perception: Experimental modification. Science, 1969, 65, 819-821.

RICHARDS, W. Motion detection in man and other animals. Brain Behavior \& Evolution, 1971, 4, 161-181.

SCOTT, T. R. \& NOLAND, J. H. Some stimulus dimensions of rotating spirals. Psychological Review, 1965, 72, 344-357.

SEKULER, R. W., \& GANZ, L. After-effect of seen motion with a stabilized retinal image. Science, 1963, 139, 419-420.

SEKULER, R. W., \& PANTLE, A. A model for after-effects of seen movement Vision Research, 1967, 7, 427-435.

SUTHERIAND, N. S. Figural after-effects and apparent size. Quarterly Joumal of Psychology, 1961, 13, 222-228.

TAYLOR, M. M. Tracking the decay of the aftereffect of seen rotary movement. Perceptual \& Motor Skills, 1963, 16, 119-129.

WALLACH, H. Informational discrepancy as a basis of perceptual adaptation. In $\mathbf{S}$. J. Freedman (Ed.), The neuropsychology of spatially oriented behavior. Homewood, Ill: Dorsey Press, 1968.

WOHLGEMUTH, A. On the after-effect of seen movement. British Journal of Psychology, Monograph Supplement, 1911, 1, 1-117.

NOTE

1. These preliminary observations were made with Dr. K. Kolehmainen at the University of Turku, Finland.

(Accepted for publication December 9 , 1971.) 